FACTA UNIVERSITATIS (NIŠ)

Ser. Math. Inform. Vol. 35, No 1 (2020), 201-215

https://doi.org/10.22190/FUMI2001201G

\title{
SOLVING THE FUZZY INITIAL VALUE PROBLEM WITH NEGATIVE COEFFICIENT BY USING FUZZY LAPLACE TRANSFORM
}

\author{
Hülya Gültekin Çitil
}

(C) 2020 by University of Niš, Serbia | Creative Commons License: CC BY-NC-ND

\begin{abstract}
In this paper, the fuzzy initial value problem with negative coefficient is solved by using fuzzy Laplace transform and generalized differentiability. The solutions are found and the comparison results are given.

Keywords: fuzzy initial value problem, generalized differentiability, fuzzy Laplace transform.
\end{abstract}

\section{Introduction}

Fuzzy differential equations have been studied by many researchers. Fuzzy differential equations can be solved by several types. Hukuhara differentiability $[10,17,23]$, generalized differentiability $[7,8,9,12]$, extension principle [10, 11], the concept of differential inclusion [16] and the fuzzy problem to be a set of crips problem [14]. Another types are numeric methods [1, 2, 3, 4, 15] and the fuzzy Laplace transform [5, 21, 22, 24].

This paper is about the solutions of the fuzzy initial value problem with negative coefficient by fuzzy Laplace transform. The aim of this study is to investigate solutions of problem using the properties fuzzy Laplace transform and generalized differentiability.

The paper is organized as follows: Section 2 delas with preliminaries, Section 3 focuses on findings and the main results, and Section 4 refers to conclusions.

\section{Preliminaries}

Definition 2.1. [20] A fuzzy number is a mapping $u: \mathbb{R} \rightarrow[0,1]$ satisfying the following properties:

$u$ is normal: $\exists x_{0} \in \mathbb{R}$ for which $u\left(x_{0}\right)=1$,

Received April 5, 2019; accepted February 11, 2020

2010 Mathematics Subject Classification. Primary 03E72; Secondary 34A07; Third 44A10 
$u$ is convex fuzzy set: $u(\lambda x+(1-\lambda) y) \geqslant \min \{u(x), u(y)\}$ for all $x, y \in \mathbb{R}, \lambda \in$ $[0,1]$,

$u$ is upper semi-continuous on $\mathbb{R}$,

$c l\{x \in \mathbb{R} \mid u(x)>0\}$ is compact, where $c l$ denotes the closure of a subset.

Let $\mathbb{R}_{F}$ denote the set of all fuzzy numbers.

Definition 2.2. [18] Let $u \in \mathbb{R}_{F}$. The $\alpha$-level set of $u$, denoted, $[u]^{\alpha}, 0<\alpha \leq 1$, is $[u]^{\alpha}=\{x \in \mathbb{R} \mid u(x) \geq \alpha\}$. If $\alpha=0,[u]^{0}=c l\{x \in \mathbb{R} \mid u(x)>0\}$. The notation, $[u]^{\alpha}=\left[\underline{u}_{\alpha}, \bar{u}_{\alpha}\right]$ denotes explicitly the $\alpha$-level set of $u$, where $\underline{u}_{\alpha}$ and $\bar{u}_{\alpha}$ denote the left-hand endpoint and the right-hand endpoint of $[u]^{\alpha}$, respectively.

The following remark shows when $\left[\underline{u}_{\alpha}, \bar{u}_{\alpha}\right]$ is a valid $\alpha$-level set.

Remark 2.1. $[13,18]$ The sufficient and necessary conditions for $\left[\underline{u}_{\alpha}, \bar{u}_{\alpha}\right]$ to define the parametric form of a fuzzy number as follows:

$\underline{u}_{\alpha}$ is bounded monotonic increasing (nondecreasing) left-continuous function on $(0,1]$ and right-continuous for $\alpha=0$,

$\bar{u}_{\alpha}$ is bounded monotonic decreasing (nonincreasing) left-continuous function on $(0,1]$ and right-continuous for $\alpha=0$,

$$
\underline{u}_{\alpha} \leq \bar{u}_{\alpha}, 0 \leq \alpha \leq 1 \text {. }
$$

Definition 2.3. [20] If A is a symmetric triangular fuzzy number with support $[\underline{a}, \bar{a}]$, the $\alpha$-level set of $A$ is $[A]^{\alpha}=\left[\underline{A}_{\alpha}, \bar{A}_{\alpha}\right]=\left[\underline{a}+\left(\frac{\bar{a}-\underline{a}}{2}\right) \alpha, \bar{a}-\left(\frac{\bar{a}-\underline{a}}{2}\right) \alpha\right]$, $\left(\underline{A}_{1}=\bar{A}_{1}, \underline{A}_{1}-\underline{A}_{\alpha}=\bar{A}_{\alpha}-\bar{A}_{1}\right)$.

Definition 2.4. $[15,18,23]$ Let $u, v \in \mathbb{R}_{F}$. If there exists $w \in \mathbb{R}_{F}$ such that $u=v+w$, then $w$ is called the Hukuhara difference of fuzzy numbers $u$ and $v$, and it is denoted by $w=u \ominus v$.

Definition 2.5. $[6,15,18]$ Let $f:[a, b] \rightarrow \mathbb{R}_{F}$ and $t_{0} \in[a, b]$. We say that $\mathrm{f}$ is Hukuhara differentiable at $t_{0}$, if there exists an element $f^{\prime}\left(t_{0}\right) \in \mathbb{R}_{F}$ such that for all $h>0$ sufficiently small, $\exists f\left(t_{0}+h\right) \ominus f\left(t_{0}\right), f\left(t_{0}\right) \ominus f\left(t_{0}-h\right)$ and the limits hold

$$
\lim _{h \rightarrow 0} \frac{f\left(t_{0}+h\right) \ominus f\left(t_{0}\right)}{h}=\lim _{h \rightarrow 0} \frac{f\left(t_{0}\right) \ominus f\left(t_{0}-h\right)}{h}=f^{\prime}\left(t_{0}\right) .
$$

Definition 2.6. [18] Let $f:[a, b] \rightarrow \mathbb{R}_{F}$ and $t_{0} \in[a, b]$. We say that $\mathrm{f}$ is (1)differentiable at $t_{0}$, if there exists an element $f^{\prime}\left(t_{0}\right) \in \mathbb{R}_{F}$ such that for all $h>0$ sufficiently small near to 0 , exist $f\left(t_{0}+h\right) \ominus f\left(t_{0}\right), f\left(t_{0}\right) \ominus f\left(t_{0}-h\right)$ and the limits

$$
\lim _{h \rightarrow 0} \frac{f\left(t_{0}+h\right) \ominus f\left(t_{0}\right)}{h}=\lim _{h \rightarrow 0} \frac{f\left(t_{0}\right) \ominus f\left(t_{0}-h\right)}{h}=f^{\prime}\left(t_{0}\right),
$$


and $\mathrm{f}$ is (2)-differentiable if for all $h>0$ sufficiently small near to 0 , exist $f\left(t_{0}\right) \ominus f\left(t_{0}+h\right), f\left(t_{0}-h\right) \ominus f\left(t_{0}\right)$ and the limits

$$
\lim _{h \rightarrow 0} \frac{f\left(t_{0}\right) \ominus f\left(t_{0}+h\right)}{-h}=\lim _{h \rightarrow 0} \frac{f\left(t_{0}-h\right) \ominus f\left(t_{0}\right)}{-h}=f^{\prime}\left(t_{0}\right) .
$$

Theorem 2.1. [19] Let $f:[a, b] \rightarrow \mathbb{R}_{F}$ be fuzzy function, where $[f(t)]^{\alpha}=$ $\left[\underline{f}_{\alpha}(t), \bar{f}_{\alpha}(t)\right]$, for each $\alpha \in[0,1]$.

(i) If $f$ is (1)-differentiable, then $\underline{f}_{\alpha}$ and $\bar{f}_{\alpha}$ are differentiable functions and $\left[f^{\prime}(t)\right]^{\alpha}=\left[\underline{f}_{\alpha}^{\prime}(t), \bar{f}_{\alpha}^{\prime}(t)\right]$,

(ii) If $f$ is (2)-differentiable, then $\underline{f}_{\alpha}$ and $\bar{f}_{\alpha}$ are differentiable functions and $\left[f^{\prime}(t)\right]^{\alpha}=\left[\bar{f}_{\alpha}^{\prime}(t), \underline{f}_{\alpha}^{\prime}(t)\right]$.

Theorem 2.2. [19] Let $f^{\prime}:[a, b] \rightarrow \mathbb{R}_{F}$ be fuzzy function, where $[f(t)]^{\alpha}=$ $\left[\underline{f}_{\alpha}(t), \bar{f}_{\alpha}(t)\right]$, for each $\alpha \in[0,1], f$ is (1)-differentiable or (2)-differentiable.

(i) If $f$ and $f$ are (1)-differentiable, then $\underline{f}_{\alpha}^{\prime}$ and $\bar{f}_{\alpha}^{\prime}$ are differentiable functions and $\left[f^{\prime \prime}(t)\right]^{\alpha}=\left[\underline{f}_{\alpha}^{\prime \prime}(t), \bar{f}_{\alpha}^{\prime \prime}(t)\right]$,

(ii) If $f$ is (1)-differentiable and $f$ is (2)-differentiable, then $\underline{f}_{\alpha}^{\prime}$ and $\bar{f}_{\alpha}^{\prime}$ are differentiable functions and $\left[f^{\prime \prime}(t)\right]^{\alpha}=\left[\bar{f}_{\alpha}^{\prime \prime}(t), \underline{f}_{\alpha}^{\prime \prime}(t)\right]$,

(iii) If $f$ is (2)-differentiable and $f$ is (1)-differentiable, then $\underline{f}_{\alpha}^{\prime}$ and $\bar{f}_{\alpha}^{\prime}$ are differentiable functions and $\left[f^{\prime \prime}(t)\right]^{\alpha}=\left[\bar{f}_{\alpha}^{\prime \prime}(t), \underline{f}_{\alpha}^{\prime \prime}(t)\right]$,

(iv) If $f$ and $f$ are (2)-differentiable, then $\underline{f}_{\alpha}^{\prime}$ and $\bar{f}_{\alpha}^{\prime}$ are differentiable functions and $\left[f^{\prime \prime}(t)\right]^{\alpha}=\left[\underline{f}_{\alpha}^{\prime \prime}(t), \bar{f}_{\alpha}^{\prime \prime}(t)\right]$.

Definition 2.7. $[22,24]$ The fuzzy Laplace transform of fuzzy-valued function $f$ is defined as follows:

$$
\begin{gathered}
F(s)=L(f(t))=\int_{0}^{\infty} e^{-s t} f(t) d t=\lim _{\tau \rightarrow \infty} \int_{0}^{\tau} e^{-s t} f(t) d t, \\
F(s)=L(f(t))=\left[\lim _{\tau \rightarrow \infty} \int_{0}^{\tau} e^{-s t} \underline{f}(t) d t, \lim _{\tau \rightarrow \infty} \int_{0}^{\tau} e^{-s t} \bar{f}(t) d t\right] . \\
F(s, \alpha)=L(f(t, \alpha))=[L(\underline{f}(t, \alpha)), L(\bar{f}(t, \alpha))]
\end{gathered}
$$


where,

$$
\begin{aligned}
& L(\underline{f}(t, \alpha))=\int_{0}^{\infty} e^{-s t} \underline{f}(t, \alpha) d t=\lim _{\tau \rightarrow \infty} \int_{0}^{\tau} e^{-s t} \underline{f}(t, \alpha) d t, \\
& L(\bar{f}(t, \alpha))=\int_{0}^{\infty} e^{-s t} \bar{f}(t, \alpha) d t=\lim _{\tau \rightarrow \infty} \int_{0}^{\tau} e^{-s t} \bar{f}(t, \alpha) d t .
\end{aligned}
$$

Theorem 2.3. [5, 22, 24] Suppose that $f$ is continuous fuzzy-valued function on $[0, \infty)$ and exponential order $\alpha$ and that $f^{\prime}$ is piecewise continuous fuzzy-valued function on $[0, \infty)$, then

$$
L\left(f^{\prime}(t)\right)=s L(f(t)) \ominus f(0),
$$

if $f$ is (1) differentiable,

$$
L\left(f^{\prime}(t)\right)=(-f(0)) \ominus(-s L(f(t)))
$$

if $f$ is (2) differentiable.

Theorem 2.4. [22, 24] Suppose that $f$ and $f$ are continuous fuzzy-valued functions on $[0, \infty)$ and of exponential order $\alpha$ and that $f^{\prime \prime}$ is piecewise continuous fuzzy-valued function on $[0, \infty)$, then

$$
L\left(f^{\prime \prime}(t)\right)=s^{2} L(f(t)) \ominus s f(0) \ominus f^{\prime}(0),
$$

if $f$ and $f$ are (1) differentiable,

$$
L\left(f^{\prime \prime}(t)\right)=-f^{\prime}(0) \ominus\left(-s^{2}\right) L(f(t))-s f(0),
$$

if $f$ is (1) differentiable and $f$ is (2) differentiable,

$$
L\left(f^{\prime \prime}(t)\right)=-s f(0) \ominus\left(-s^{2}\right) L(f(t)) \ominus f^{\prime}(0),
$$

if $f$ is (2) differentiable and $f$ is (1) differentiable,

$$
L\left(f^{\prime \prime}(t)\right)=s^{2} L(f(t)) \ominus s f(0)-f^{\prime}(0),
$$

if $f$ and $f$ are (2) differentiable.

Theorem 2.5. [5, 22] Let $f(x), g(x)$ be continuous fuzzy-valued functions suppose that $c_{1}$ and $c_{2}$ are constant, then

$$
L\left(c_{1} f(x)+c_{2} g(x)\right)=\left(c_{1} L(f(x))\right)+\left(c_{2} L(g(x))\right) .
$$

Theorem 2.6. [5] Let $f(x)$ be continuous fuzzy-valued function on $[0, \infty)$ and $\lambda \geq 0$, then

$$
L(\lambda f(x))=\lambda(L(f(x)))
$$




\section{Findings and Main Results}

In this section, we consider solutions of the fuzzy initial value problem

$$
\begin{gathered}
y^{\prime \prime}(t)=-\lambda y(t), t>0, \\
y(0)=[A]^{\alpha}, \quad y^{\prime}(0)=[B]^{\alpha},
\end{gathered}
$$

by Laplace transform, where $\lambda>0, \mathrm{~A}$ and $\mathrm{B}$ are symmetric triangular fuzzy numbers with supports $[\underline{a}, \bar{a}]$ and $[\underline{b}, \bar{b}]$, respectively,

$$
\begin{gathered}
{[A]^{\alpha}=\left[\underline{A}_{\alpha}, \bar{A}_{\alpha}\right]=\left[\underline{a}+\left(\frac{\bar{a}-\underline{a}}{2}\right) \alpha, \bar{a}-\left(\frac{\bar{a}-\underline{a}}{2}\right) \alpha\right],} \\
{[B]^{\alpha}=\left[\underline{B}_{\alpha}, \bar{B}_{\alpha}\right]=\left[\underline{b}+\left(\frac{\bar{b}-\underline{b}}{2}\right) \alpha, \bar{b}-\left(\frac{\bar{b}-\underline{b}}{2}\right) \alpha\right],}
\end{gathered}
$$

$(\mathrm{i}, \mathrm{j})$ solution means that $\mathrm{y}$ is (i) differentiable, $y^{\prime}$ is $(\mathrm{j})$ differentiable.

Case 1) If $y$ and $y^{\prime}$ are (1) differentiable, since

$$
s^{2} L(y(t, \alpha)) \ominus s y(0, \alpha) \ominus y^{\prime}(0, \alpha)=-\lambda L(y(t, \alpha)),
$$

we have the equations

$$
\begin{aligned}
& s^{2} L(\underline{y}(t, \alpha))-s \underline{y}(0, \alpha)-\underline{y}^{\prime}(0, \alpha)=-\lambda \bar{y}(t, \alpha), \\
& s^{2} L(\bar{y}(t, \alpha))-s \bar{y}(0, \alpha)-\bar{y}^{\prime}(0, \alpha)=-\lambda \underline{y}(t, \alpha) .
\end{aligned}
$$

Using the initial values and taking the necessary operations,

$$
\begin{aligned}
& L(\underline{y}(t, \alpha))=\frac{s^{2}}{s^{4}-\lambda^{2}} \underline{B}_{\alpha}+\frac{s^{3}}{s^{4}-\lambda^{2}} \underline{A}_{\alpha}-\frac{\lambda s}{s^{4}-\lambda^{2}} \bar{A}_{\alpha}-\frac{\lambda}{s^{4}-\lambda^{2}} \bar{B}_{\alpha}, \\
& L(\bar{y}(t, \alpha))=\frac{s^{2}}{s^{4}-\lambda^{2}} \bar{B}_{\alpha}+\frac{s^{3}}{s^{4}-\lambda^{2}} \bar{A}_{\alpha}-\frac{\lambda s}{s^{4}-\lambda^{2}} \underline{A}_{\alpha}-\frac{\lambda}{s^{4}-\lambda^{2}} \underline{B}_{\alpha} .
\end{aligned}
$$

From here,

$$
\begin{aligned}
\underline{y}(t, \alpha)= & L^{-1}\left(\frac{s^{2}}{s^{4}-\lambda^{2}}\right) \underline{B}_{\alpha}+L^{-1}\left(\frac{s^{3}}{s^{4}-\lambda^{2}}\right) \underline{A}_{\alpha} \\
& -L^{-1}\left(\frac{\lambda s}{s^{4}-\lambda^{2}}\right) \bar{A}_{\alpha}-L^{-1}\left(\frac{\lambda}{s^{4}-\lambda^{2}}\right) \bar{B}_{\alpha},
\end{aligned}
$$




$$
\begin{aligned}
\bar{y}(t, \alpha)= & L^{-1}\left(\frac{s^{2}}{s^{4}-\lambda^{2}}\right) \bar{B}_{\alpha}+L^{-1}\left(\frac{s^{3}}{s^{4}-\lambda^{2}}\right) \bar{A}_{\alpha} \\
& -L^{-1}\left(\frac{\lambda s}{s^{4}-\lambda^{2}}\right) \underline{A}_{\alpha}-L^{-1}\left(\frac{\lambda}{s^{4}-\lambda^{2}}\right) \underline{B}_{\alpha}
\end{aligned}
$$

Thus, the lower solution and the upper solution are obtained as

$$
\begin{aligned}
\underline{y}(t, \alpha)= & \frac{e^{\sqrt{\lambda} t}}{4}\left(\frac{\underline{B}_{\alpha}-\bar{B}_{\alpha}}{\sqrt{\lambda}}+\underline{A}_{\alpha}-\bar{A}_{\alpha}\right) \\
& +\frac{e^{-\sqrt{\lambda} t}}{4}\left(\frac{\bar{B}_{\alpha}-\underline{B}_{\alpha}}{\sqrt{\lambda}}+\underline{A}_{\alpha}-\bar{A}_{\alpha}\right) \\
& +\frac{\sin (\sqrt{\lambda} t)}{2 \sqrt{\lambda}}\left(\underline{B}_{\alpha}+\bar{B}_{\alpha}\right)+\frac{\cos (\sqrt{\lambda} t)}{2}\left(\underline{A}_{\alpha}+\bar{A}_{\alpha}\right), \\
\bar{y}(t, \alpha)= & \frac{e^{\sqrt{\lambda} t}\left(\frac{\bar{B}_{\alpha}-\underline{B}_{\alpha}}{4}+\bar{A}_{\alpha}-\underline{A}_{\alpha}\right)}{\sqrt{\lambda}}+\frac{e^{-\sqrt{\lambda} t}\left(\frac{\underline{B}_{\alpha}-\bar{B}_{\alpha}}{\sqrt{\lambda}}+\bar{A}_{\alpha}-\underline{A}_{\alpha}\right)}{4} \\
& +\frac{\sin (\sqrt{\lambda} t)}{2 \sqrt{\lambda}}\left(\underline{B}_{\alpha}+\bar{B}_{\alpha}\right)+\frac{\cos (\sqrt{\lambda} t)}{2}\left(\underline{A}_{\alpha}+\bar{A}_{\alpha}\right) .
\end{aligned}
$$

Case 2) If $y$ is (1) differentiable and $y^{\prime}$ is (2) differentiable, since

$$
-y^{\prime}(0, \alpha) \ominus\left(-s^{2}\right) L(y(t, \alpha))-s y(0, \alpha)=-\lambda L(y(t, \alpha)),
$$

we have the equations

$$
\begin{aligned}
& -\bar{y}^{\prime}(0, \alpha)-\left(-s^{2} L(\bar{y}(t, \alpha))\right)-s \bar{y}(0, \alpha)=-\lambda L(\bar{y}(t, \alpha)), \\
& -\underline{y}^{\prime}(0, \alpha)-\left(-s^{2} L(\underline{y}(t, \alpha))\right)-s \underline{y}(0, \alpha)=-\lambda L(\underline{y}(t, \alpha)) .
\end{aligned}
$$

Using the initial values, we get

$$
\begin{aligned}
& L(\underline{y}(t, \alpha))=\frac{1}{s^{2}+\lambda} \underline{B}_{\alpha}+\frac{s}{s^{2}+\lambda} \underline{A}_{\alpha} . \\
& L(\bar{y}(t, \alpha))=\frac{1}{s^{2}+\lambda} \bar{B}_{\alpha}+\frac{s}{s^{2}+\lambda} \bar{A}_{\alpha} .
\end{aligned}
$$

Taking inverse Laplace transforms of the equations, we have

$$
\underline{y}(t, \alpha)=L^{-1}\left(\frac{1}{s^{2}+\lambda}\right) \underline{B}_{\alpha}+L^{-1}\left(\frac{s}{s^{2}+\lambda}\right) \underline{A}_{\alpha}
$$




$$
\bar{y}(t, \alpha)=L^{-1}\left(\frac{1}{s^{2}+\lambda}\right) \bar{B}_{\alpha}+L^{-1}\left(\frac{s}{s^{2}+\lambda}\right) \bar{A}_{\alpha} .
$$

From this, the lower and the upper solutions are obtained as

$$
\begin{aligned}
& \underline{y}(t, \alpha)=\frac{1}{\sqrt{\lambda}} \sin (\sqrt{\lambda} t) \underline{B}_{\alpha}+\cos (\sqrt{\lambda} t) \underline{A}_{\alpha}, \\
& \bar{y}(t, \alpha)=\frac{1}{\sqrt{\lambda}} \sin (\sqrt{\lambda} t) \bar{B}_{\alpha}+\cos (\sqrt{\lambda} t) \bar{A}_{\alpha} .
\end{aligned}
$$

Case 3) If $y$ is (2) differentiable and $y^{\prime}$ is (1) differentiable, since

$$
-s y(0, \alpha) \ominus\left(-s^{2}\right) L(y(t, \alpha)) \ominus y^{\prime}(0, \alpha)=-\lambda L(y(t, \alpha))
$$

we have the equations

$$
\begin{gathered}
-s \bar{y}(0, \alpha)-\left(-s^{2} L(\bar{y}(t, \alpha))\right)-\bar{y}^{\prime}(0, \alpha)=-\lambda L(\bar{y}(t, \alpha)) \\
-s \underline{y}(0, \alpha)-\left(-s^{2} L(\underline{y}(t, \alpha))\right)-\underline{y}^{\prime}(0, \alpha)=-\lambda L(\underline{y}(t, \alpha)) .
\end{gathered}
$$

Using the initial values and taking inverse Laplace transforms of the equations, we have

$$
\begin{aligned}
& \underline{y}(t, \alpha)=\frac{1}{\sqrt{\lambda}} \sin (\sqrt{\lambda} t) \bar{B}_{\alpha}+\cos (\sqrt{\lambda} t) \underline{A}_{\alpha} . \\
& \bar{y}(t, \alpha)=\frac{1}{\sqrt{\lambda}} \sin (\sqrt{\lambda} t) \underline{B}_{\alpha}+\cos (\sqrt{\lambda} t) \bar{A}_{\alpha} .
\end{aligned}
$$

Case 4) If $y$ is (2) differentiable and $y^{\prime}$ is (2) differentiable, since

$$
s^{2} L(y(t, \alpha)) \ominus s y(0, \alpha)-y^{\prime}(0, \alpha)=-\lambda L(y(t, \alpha))
$$

we have the equations

$$
\begin{aligned}
& s^{2} L(\underline{y}(t, \alpha))-s \underline{y}(0, \alpha)-\underline{y}^{\prime}(0, \alpha)=-\lambda L(\bar{y}(t, \alpha)) \\
& s^{2} L(\bar{y}(t, \alpha))-s \bar{y}(0, \alpha)-\bar{y}^{\prime}(0, \alpha)=-\lambda L(\underline{y}(t, \alpha))
\end{aligned}
$$

Using the initial values and taking the necessary operations,

$$
L(\underline{y}(t, \alpha))=\frac{s^{3}}{s^{4}-\lambda^{2}} \underline{A}_{\alpha}+\frac{s^{2}}{s^{4}-\lambda^{2}} \bar{B}_{\alpha}-\frac{\lambda s}{s^{4}-\lambda^{2}} \bar{A}_{\alpha}-\frac{\lambda}{s^{4}-\lambda^{2}} \underline{B}_{\alpha},
$$




$$
L(\bar{y}(t, \alpha))=\frac{s^{3}}{s^{4}-\lambda^{2}} \bar{A}_{\alpha}+\frac{s^{2}}{s^{4}-\lambda^{2}} \underline{B}_{\alpha}-\frac{\lambda s}{s^{4}-\lambda^{2}} \underline{A}_{\alpha}-\frac{\lambda}{s^{4}-\lambda^{2}} \bar{B}_{\alpha} .
$$

Taking inverse Laplace transforms of the equations, the lower and the upper solutions are obtained as

$$
\begin{aligned}
\underline{y}(t, \alpha)= & \frac{e^{\sqrt{\lambda} t}}{4}\left(\frac{\bar{B}_{\alpha}-\underline{B}_{\alpha}}{\sqrt{\lambda}}+\underline{A}_{\alpha}-\bar{A}_{\alpha}\right) \\
& +\frac{e^{-\sqrt{\lambda} t}}{4}\left(\frac{\underline{B}_{\alpha}-\bar{B}_{\alpha}}{\sqrt{\lambda}}+\underline{A}_{\alpha}-\bar{A}_{\alpha}\right) \\
& +\frac{\sin (\sqrt{\lambda} t)}{2 \sqrt{\lambda}}\left(\underline{B}_{\alpha}+\bar{B}_{\alpha}\right)+\frac{\cos (\sqrt{\lambda} t)}{2}\left(\underline{A}_{\alpha}+\bar{A}_{\alpha}\right), \\
\bar{y}(t, \alpha)= & \frac{e^{\sqrt{\lambda} t}}{4}\left(\frac{\underline{B}_{\alpha}-\bar{B}_{\alpha}}{\sqrt{\lambda}}+\bar{A}_{\alpha}-\underline{A}_{\alpha}\right) \\
& +\frac{e^{-\sqrt{\lambda} t}}{4}\left(\frac{\bar{B}_{\alpha}-\underline{B}_{\alpha}}{\sqrt{\lambda}}+\bar{A}_{\alpha}-\underline{A}_{\alpha}\right) \\
& +\frac{\sin (\sqrt{\lambda} t)}{2 \sqrt{\lambda}}\left(\underline{B}_{\alpha}+\bar{B}_{\alpha}\right)+\frac{\cos (\sqrt{\lambda} t)}{2}\left(\underline{A}_{\alpha}+\bar{A}_{\alpha}\right) .
\end{aligned}
$$

Theorem 3.1. (1,1) solution of the initial value problem (3.1)-(3.2) is a valid $\alpha$-level set for $t>0$ satisfying the inequality

$$
e^{2 \sqrt{\lambda} t} \geq\left(\frac{(\bar{b}-\underline{b})-\sqrt{\lambda}(\bar{a}-\underline{a})}{(\bar{b}-\underline{b})+\sqrt{\lambda}(\bar{a}-\underline{a})}\right) .
$$

Proof. If

$$
\frac{\partial \underline{y}(t, \alpha)}{\partial \alpha} \geq 0, \quad \frac{\partial \bar{y}(t, \alpha)}{\partial \alpha} \leq 0, \quad \underline{y}(t, \alpha) \leq \bar{y}(t, \alpha)
$$

$(1,1)$ solution of the initial value problem $(3.1)-(3.2)$ is valid $\alpha$-level set. Thus, it must be

$$
e^{\sqrt{\lambda} t}\left(\frac{\bar{b}-\underline{b}}{\sqrt{\lambda}}+\bar{a}-\underline{a}\right)-e^{-\sqrt{\lambda} t}\left(\frac{\bar{b}-\underline{b}}{\sqrt{\lambda}}-\bar{a}-\underline{a}\right) \geq 0
$$

Since

$$
(\bar{b}-\underline{b})+\sqrt{\lambda}(\bar{a}-\underline{a}) \geq 0,
$$

we have

$$
e^{\sqrt{\lambda} t} \geq e^{-\sqrt{\lambda} t}\left(\frac{(\bar{b}-\underline{b})-\sqrt{\lambda}(\bar{a}-\underline{a})}{(\bar{b}-\underline{b})+\sqrt{\lambda}(\bar{a}-\underline{a})}\right)
$$


Consequently, $(1,1)$ solution of the initial value problem (3.1)-(3.2) is a valid $\alpha$-level set for $t>0$ satisfying the inequality

$$
e^{2 \sqrt{\lambda} t} \geq\left(\frac{(\bar{b}-\underline{b})-\sqrt{\lambda}(\bar{a}-\underline{a})}{(\bar{b}-\underline{b})+\sqrt{\lambda}(\bar{a}-\underline{a})}\right) .
$$

Theorem 3.2. (1,2) solution of the initial value problem (3.1)-(3.2) is valid $\alpha$-level set, for $t \in\left(0, \frac{\pi}{2 \sqrt{\lambda}}\right)$ satisfying the inequality

$$
t \geq \frac{1}{\sqrt{\lambda}} \tan ^{-1}\left(-\sqrt{\lambda}\left(\frac{\bar{a}-\underline{a}}{\bar{b}-\underline{b}}\right)\right) .
$$

Proof. If

$$
\frac{1}{\sqrt{\lambda}} \sin (\sqrt{\lambda} t)(\bar{b}-\underline{b})+\cos (\sqrt{\lambda} t)(\bar{a}-\underline{a}) \geq 0
$$

$(1,2)$ solution of the initial value problem (3.1)-(3.2) is valid $\alpha$-level set. For $\sqrt{\lambda} t \in$ $\left(0, \frac{\pi}{2}\right) \Rightarrow t \in\left(0, \frac{\pi}{2 \sqrt{\lambda}}\right)$, we have

$$
\tan (\sqrt{\lambda} t) \geq-\sqrt{\lambda}\left(\frac{\bar{a}-\underline{a}}{\bar{b}-\underline{b}}\right) \Rightarrow t \geq \frac{1}{\sqrt{\lambda}} \tan ^{-1}\left(-\sqrt{\lambda}\left(\frac{\bar{a}-\underline{a}}{\bar{b}-\underline{b}}\right)\right) .
$$

This completes the proof.

Theorem 3.3. (2,1) solution of the initial value problem (3.1)-(3.2) is a valid $\alpha$-level set for $t \in\left(0, \frac{\pi}{2 \sqrt{\lambda}}\right)$ satisfying the inequality

$$
t \leq \frac{1}{\sqrt{\lambda}} \tan ^{-1}\left(\sqrt{\lambda}\left(\frac{\bar{a}-\underline{a}}{\bar{b}-\underline{b}}\right)\right) .
$$

Proof. The proof is similar.

Theorem 3.4. (2,2) solution of the initial value problem (3.1)-(3.2) is a valid $\alpha$-level set for $t>0$ satisfying the inequality

$$
e^{-2 \sqrt{\lambda} t} \geq\left(\frac{(\bar{b}-\underline{b})-\sqrt{\lambda}(\bar{a}-\underline{a})}{(\bar{b}-\underline{b})+\sqrt{\lambda}(\bar{a}-\underline{a})}\right) .
$$

Proof. The proof is similar.

Theorem 3.5. All of the solutions are symmetric triangular fuzzy numbers for any $t>0$. 
Proof. For $(1,1)$ solution, since

$$
\underline{y}(t, 1)=\frac{\sin (\sqrt{\lambda t})}{2 \sqrt{\lambda}}(\bar{b}+\underline{b})+\frac{\cos (\sqrt{\lambda t})}{2}(\bar{a}+\underline{a})=\bar{y}(t, 1),
$$

and

$$
\begin{aligned}
\underline{y}(t, 1)-\underline{y}(t, \alpha)= & \frac{e^{\sqrt{\lambda t}}}{4}(1-\alpha)\left(\frac{\bar{b}-\underline{b}}{\sqrt{\lambda}}+(\bar{a}-\underline{a})\right) \\
& \frac{e^{-\sqrt{\lambda t}}}{4}(\alpha-1)\left(\frac{\bar{b}-\underline{b}}{\sqrt{\lambda}}-(\bar{a}-\underline{a})\right) \\
= & \bar{y}(t, \alpha)-\bar{y}(t, 1),
\end{aligned}
$$

$(1,1)$ solution of the initial value problem (3.1)-(3.2) is a symmetric triangular fuzzy number for any $t>0$. For $(1,2)$ solution, since

$$
\underline{y}(t, 1)=\frac{1}{\sqrt{\lambda}} \sin (\sqrt{\lambda t})\left(\frac{\bar{b}+\underline{b}}{2}\right)+\cos (\sqrt{\lambda t})\left(\frac{\bar{a}+\underline{a}}{2}\right)=\bar{y}(t, 1),
$$

and

$$
\begin{aligned}
\underline{y}(t, 1)-\underline{y}(t, \alpha) & =(1-\alpha)\left(\frac{1}{\sqrt{\lambda}} \sin (\sqrt{\lambda t})\left(\frac{\bar{b}-\underline{b}}{2}\right)+\cos (\sqrt{\lambda t})\left(\frac{\bar{a}-\underline{a}}{2}\right)\right) \\
& =\bar{y}(t, \alpha)-\bar{y}(t, 1),
\end{aligned}
$$

$(1,2)$ solution of the initial value problem (3.1)-(3.2) is a symmetric triangular fuzzy number for any $t>0$. For $(1,2)$ and $(2,2)$ solutions, the proof is similar.

Example 3.1. Consider the solutions of the fuzzy initial value problem

$$
y^{\prime \prime}(t)=-y(t), \quad y(0)=[1]^{\alpha}, \quad y^{\prime}(0)=[2]^{\alpha}
$$

by fuzzy Laplace transform, where $[1]^{\alpha}=[\alpha, 2-\alpha],[2]^{\alpha}=[1+\alpha, 3-\alpha]$ with supports $[0,2]$ and $[1,3]$, respectively.

For $(1,1)$ solution, the lower and the upper solutions are

$$
\begin{aligned}
& \underline{y}(t, \alpha)=e^{t}(\alpha-1)+2 \sin t+\cos t, \\
& \bar{y}(t, \alpha)=e^{t}(1-\alpha)+2 \sin t+\cos t .
\end{aligned}
$$

For $(1,2)$ solution, the lower and the upper solutions are

$$
\begin{gathered}
\underline{y}(t, \alpha)=(1+\alpha) \sin (t)+\alpha \cos (t), \\
\bar{y}(t, \alpha)=(3-\alpha) \sin (t)+(2-\alpha) \cos (t) .
\end{gathered}
$$

For $(2,1)$ solution, the lower and the upper solutions are

$$
\underline{y}(t, \alpha)=(3-\alpha) \sin (t)+\alpha \cos (t),
$$




$$
\bar{y}(t, \alpha)=(1+\alpha) \sin (t)+(2-\alpha) \cos (t) .
$$

For $(2,2)$ solution, the lower and the upper solutions are

$$
\begin{aligned}
& \underline{y}(t, \alpha)=e^{-t}(\alpha-1)+2 \sin t+\cos t, \\
& \bar{y}(t, \alpha)=e^{-t}(1-\alpha)+2 \sin t+\cos t .
\end{aligned}
$$

$(1,1)$ solution is a valid $\alpha$-level set since $e^{t}>0$. (1,2) solution is a valid $\alpha$-level set since $t-\tan ^{-1}(-1)>0$ for $t \in\left(0, \frac{\pi}{2}\right)$ according to figure 3.1. $(2,1)$ solution is a valid $\alpha$-level set since $t-\tan ^{-1}(1) \leq 0$ for $t \in(0,0.785398]$ on $\left(0, \frac{\pi}{2}\right)$ according to figure 3.2. (2,2) solution is a valid $\alpha$-level set since $e^{-t}>0$. All of the solutions are symmetric triangular fuzzy numbers. Also, we can see graphics of solutions for $\alpha=0.2$ in figure 3.3-figure 3.6.

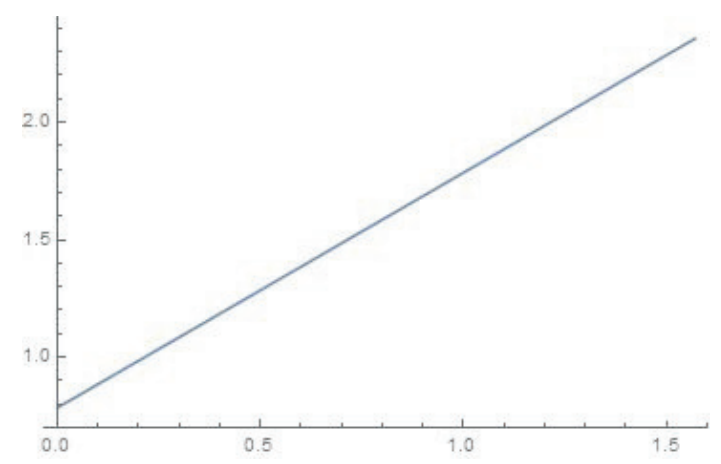

Figure 3.1. Graphic of the function $t-\tan ^{-1}(-1)$

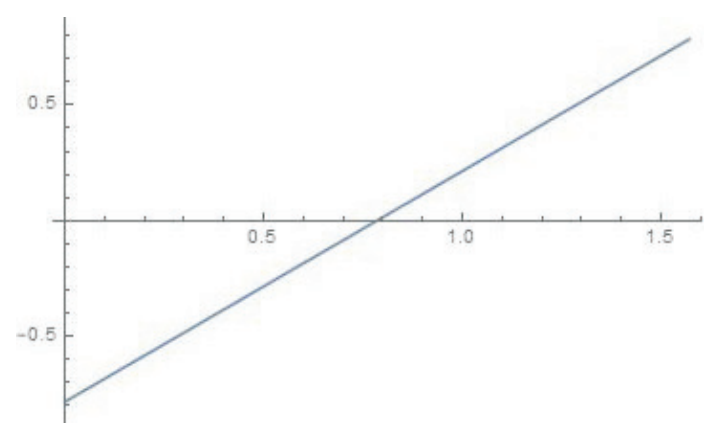

Figure 3.2. Graphic of the function $t-\tan ^{-1}(1)$ 


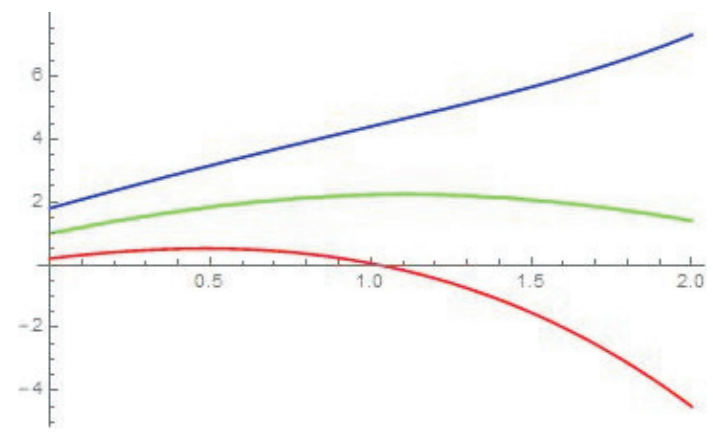

Figure 3.3. Graphic of $(1,1)$ solution for $\alpha=0.2$

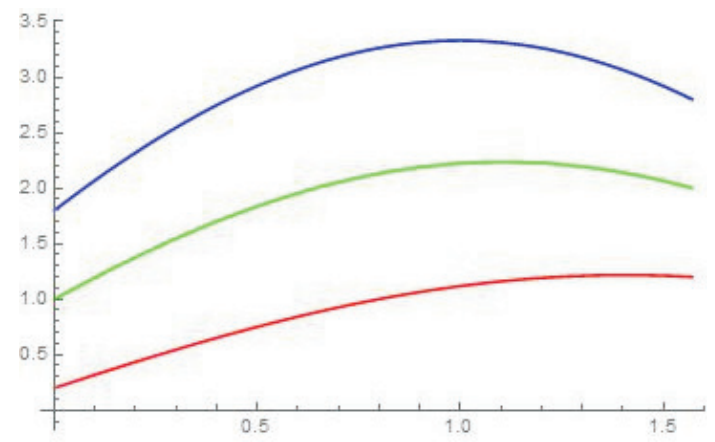

Figure 3.4. Graphic of $(1,2)$ solution for $\alpha=0.2$

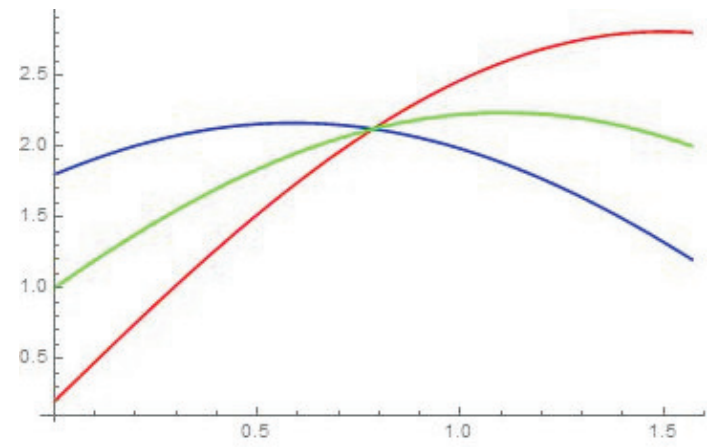

Figure 3.5. Graphic of $(2,1)$ solution for $\alpha=0.2$ 


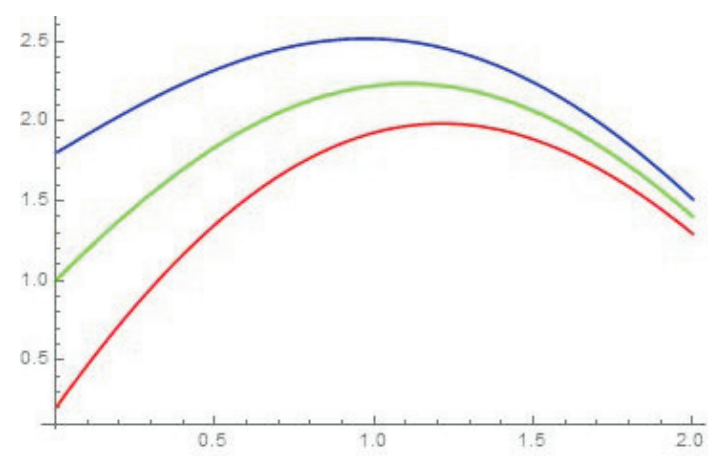

Figure 3.6. Graphic of $(2,2)$ solution for $\alpha=0.2$

$$
\text { Red } \rightarrow \underline{y}_{\alpha}(t), \text { Blue } \rightarrow \bar{y}_{\alpha}(t), \text { Green } \rightarrow \underline{y}_{1}(t)=\bar{y}_{1}(t)
$$

\section{Conclusions}

In this paper, fuzzy initial value problem with negative coefficient is studied by fuzzy Laplace transform and generalized differentiability. Solutions are found and comparison results are given. It has been proved that the solutions are valid fuzzy functions, which has been shown on an example. It has also been found that when $(1,1),(2,1)$ and $(2,2)$ solutions are valid $\alpha$ - level sets, $(1,2)$ solution is a valid $\alpha-$ level set for $t \in(0,0.785398]$. However, we can see that $(1,1)$ solution behaves differently from the crips solution in figure 3.3 . It means that $(1,1)$ solution becomes fuzzier as time goes by.

\section{REF E R E N C E S}

1. S. AbBasbandy and T. Allahviranloo: Numerical solutions of fuzzy differential equations by Taylor method. Computational Methods in Applied Mathematics 2:2 (2002), 113-124.

2. S. Abbasbandy, T. Allahviranloo, O. Lopez-Pouso and J. J. Nieto: Numerical methods for fuzzy differential inclusions. Computers and Mathematics with Applications 48:10-11 (2004), 1633-1641.

3. T. Allahviranloo, N. Ahmady and E. Ahmady: Numerical solution of fuzzy differential equations by predictor-corrector method. Information Sciences 177:7 (2007), 1633-1647.

4. T. Allahviranloo, E. Ahmady and N. Ahmady : Nth-order fuzzy linear differential equations. Information Sciences 178:5 (2008), 1309-1324.

5. T. Allahviranloo and M. Barkhordari Ahmadi: Fuzzy Laplace transforms. Soft Computing 14:3 (2010), 235-243.

6. B. BEDE: Note on "Numerical solution of fuzzy differential equations by predictorcorrector method". Information Sciences 178:7 (2008), 1917-1922. 
7. B. BedE and S. G. GAL: Almost periodic fuzzy-number-valued functions. Fuzzy Sets and Systems 147:3 (2004), 385-403.

8. B. BEDE and S. G. GAL: Generalizations of the differentiability of fuzzy-number-valued functions with applications to fuzzy differential equations. Fuzzy Sets and Systems 151:3 (2005), 581-599.

9. B. Bede, I. J. RudAs and A. L. BenCsiK: First order linear fuzzy differential equations under generalized differentiability. Information Sciences 177:7 (2007), 1648-1662.

10. J. J. Buckley and T. Feuring: Fuzzy differential equations. Fuzzy Sets and Systems 110:1 (2000), 43-54.

11. J. J. Buckley and T. Feuring: Fuzzy initial value problem for Nth-order linear differential equations. Fuzzy Sets and Systems 121:2 (2001), 247-255.

12. Y. Chalco-Cano and H. Roman-Flores: On new solutions of fuzzy differential equations. Chaos, Solitons and Fractals 38:1 (2008), 112-119.

13. D. Dubois and H. Prade: Operations on fuzzy numbers. International Journal of Systems Science 9:6 (1978), 613-626.

14. N. Gasilov, S. E. Amrahov and A. G. Fatullayev: A geometric approach to solve fuzzy linear systems of differential equations. Applied Mathematics and Information Sciences 5:3 (2011), 484-499.

15. X. Guo, D. Shang and X. LU: Fuzzy approximate solutions of second-order fuzzy linear boundary value problems. Boundary Value Problems 2013:212 (2013), 1-17.

16. H. HÜLLERMEIER: An approach to modelling and simulation of uncertain dynamical systems. International Journal of Uncertainty, Fuzziness and Knowledge-Based Systems. 5:2 (1997), 117-137.

17. O. Kaleva: Fuzzy differential equations. Fuzzy Sets and Systems 24:3 (1987), 301317.

18. A. Khastan and J. J. Nieto: A boundary value problem for second order fuzzy differential equations. Nonlinear Analysis 72:9-10 (2010), 3583-3593.

19. A. Khastan, F. Bahrami and K. Ivaz: New results on multiple solutions for Nthorder fuzzy differential equations under generalized differentiability. Boundary Value Problems (2009), doi:10.1155/2009/395714, 1-13.

20. H-K. LIU: Comparison results of two-point fuzzy boundary value problems. International Journal of Computational and Mathematical Sciences. 5:1 (2011), 1-7.

21. K. R. Patel and N. B. Desai: Solution of fuzzy initial value problems by fuzzy Laplace transform. Kalpa Publications in Computing 2 (2017), 25-37.

22. K. R. PATEL and N. B. Desai: Solution of variable coefficient fuzzy differential equations by fuzzy Laplace transform. International Journal on Recent and Innovation Trends in Computing and Communication. 5:6 (2017), 927-942.

23. M. L. Puri and D. A. RAlescu: Differentials for fuzzy functions. Journal of Mathematical Analysis and Applications 91:2 (1983), 552-558.

24. S. Salahshour and T. Allahviranloo: Applications of fuzzy Laplace transforms. Soft Computing 17:1 (2013), 145-158.

Hülya Gültekin Çitil

Department of Mathematics 
Faculty of Arts and Science

Giresun University

Giresun, Turkey

hulya.citil@giresun.edu.tr 